\title{
ZERO-ONE LAWS FOR NON-GAUSSIAN MEASURES
}

\author{
JOEL ZINN $^{1}$
}

\begin{abstract}
Some zero-one laws are proved for non-Gaussian measures on the space $\boldsymbol{R}^{\infty}$. Also included is a characterization of the generating Hilbert space of an abstract Wiener space in terms of the subgroups of positive measure.
\end{abstract}

1. Introduction. In their 1970 paper [4] B. Jamison and S. Orey proved the following result: If $\mu$ is the product normalized Gaussian measure on the space $\left(\boldsymbol{R}^{\infty}, \mathscr{B}^{\infty}\right)$ and $G$ is a completion measurable subgroup of $\boldsymbol{R}^{\infty}$, then $\mu(G)=0$ or 1 . They then applied this result to extend a theorem of R. H. Cameron and R. E. Graves [1]. Namely, they prove the following.

TheOREM. Let $\mu$ be a mean-zero Gaussian measure on the space $C(I)$ of continuous functions on the unit interval. If $G$ is a completion measurable subgroup of $C(I)$, then $\mu(G)=0$ or 1 .

Using different techniques G. Kallianpur [6] showed that the result holds for Gaussian measures with continuous covariance functions on a measurable space $(X, \mathscr{B}(X))$, where $X$ is a linear space of real-valued functions over a complete separable metric space, and $\mathscr{B}(X)$ is the $\sigma$ algebra generated by the cylinder sets. However, Kallianpur's result holds only for completion measurable $r$-modules and $\mathscr{B}(X)$ measurable subgroups. In 1971 N. Jain [2] showed that Kallianpur's result holds for completion measurable subgroups.

In $\S 3$ we continue this investigation for certain non-Gaussian measures on $\left(\boldsymbol{R}^{\infty}, \mathscr{B}^{\infty}\right)$ and we apply this to measures on topological vector spaces. In $\S 4$ we give a characterization of the generating Hilbert space of an abstract Wiener space in terms of the measurable subgroups of positive measure, and we also give some examples.

2. Notation and preliminaries. In this section $\boldsymbol{R}^{\infty}$ is the countable product of the real line with the product topology, and $\mathscr{B}{ }^{\infty}$ is the $\sigma$-field of Borel subsets of $\boldsymbol{R}^{\infty}$. For a probability measure $\mu$ on $\boldsymbol{R}^{\infty}, \mathscr{B}_{\mu}^{\infty}$ will denote the $\mu$-completion of $\mathscr{B}{ }^{\infty}$.

Received by the editors May 4, 1973.

AMS (MOS) subject classifications (1970). Primary 60B05, 60B15.

Key words and phrases. Zero-one law, non-Gaussian measure.

${ }^{1}$ Partially supported by NSF Grant 28683. 
Let $\pi_{n}: \boldsymbol{R}^{\infty} \rightarrow \boldsymbol{R}^{\infty}$ be defined by $\pi_{n}\left(x_{1}, x_{2}, \cdots\right)=\left(x_{1}, \cdots, x_{n}, 0,0 \cdots\right)$, and let $\boldsymbol{R}^{n}=\pi_{n}\left(\boldsymbol{R}^{\infty}\right) . \boldsymbol{R}^{n}$ will be given the subspace topology (the usual Euclidean topology) and $\mathscr{B}^{n}$ will denote the associated Borel $\sigma$-field. Lebesgue measure on $\boldsymbol{R}^{n}$ will be written as $m^{(n)}$. For a probability measure $\mu$ on $\left(\boldsymbol{R}^{\infty}, \mathscr{B}^{\infty}\right)$ and $\varphi$ a measurable mapping of $\left(\boldsymbol{R}^{\infty}, \mathscr{B}^{\infty}\right)$ to the measurable space $(Y, \mathscr{C})$, we define the probability measure $\mu^{\varphi}$ on $(Y, \mathscr{C})$ by $\mu^{\varphi}(C)=\mu\left(\varphi^{-1}(C)\right)$ for all $C \in \mathscr{C}$. In the case $\varphi(x)=x+y$ for some $y \in \boldsymbol{R}^{\infty}$, $\mu^{\varphi}$ will be written simply as $\mu_{y}$, i.e., $\mu_{y}(E)=\mu(E-y)$.

For subgroups $H$ and $G$ of $\boldsymbol{R}^{\infty}, H \leqq G$ will mean that $H$ is a subgroup of $G$. For $S \subseteq R^{\infty},\langle S\rangle$ will mean the group generated by $S$. Of particular importance to us is the subgroup $\bigcup_{n=1}^{\infty} \boldsymbol{R}^{n}$, which will be denoted by $l_{0}$.

Finally, let $\mathscr{M}$ be the set of probability measures on $\left(\boldsymbol{R}^{\infty}, \mathscr{B}^{\infty}\right)$ and define:

$$
\begin{aligned}
& \mathscr{E}_{1}=\left\{\mu \in \mathscr{M}: G \in \mathscr{B}_{\mu}^{\infty}, G \leqq \boldsymbol{R}^{\infty}, \mu(G)>0 \Rightarrow G \geqq l_{0}\right\}, \\
& \mathscr{E}_{2}=\left\{\mu \in \mathscr{M}: \mu^{\pi}{ }{ }^{(n)} \text { for all } n\right\}, \\
& \mathscr{E}_{3}=\left\{\mu \in \mathscr{M}: \text { given any compact set } K \subseteq \boldsymbol{R}^{\infty}, N\right. \text { a positive } \\
& \text { integer, and }\left\{x_{i}\right\} \text { a sequence in } \boldsymbol{R}^{N} \text { converging to } \\
&\left.\quad 0, \text { we have } \mu\left(K-x_{i}\right) \rightarrow \mu(K)\right\},
\end{aligned}
$$

and

$\mathscr{P}=\left\{\mu \in \mathscr{E}_{2}: \mu=\prod_{i=1}^{\infty} \mu_{i}\right.$, where $\mu_{i}$ is a probability measure on the real line $\}$.

3. In this section we describe certain properties of the collections $\mathscr{E}_{i}$ and $\mathscr{P}$, and show how the study of these collections relates to the zeroone law for subgroups.

THEOREM 1. $\mathscr{E}_{3} \subseteq \mathscr{E}_{1}$.

Proof. Assume that $\mu \in \mathscr{E}_{3}$ and that $G$ is a $\mathscr{B}^{\infty}$-measurable subgroup of $\boldsymbol{R}^{\infty}$ with $\mu(G)>0$. Let $\left\{z_{\alpha}: \alpha \in A\right\}$ be a complete set of coset representatives for $G$ in $\boldsymbol{R}^{\infty}$, i.e., $\left\{G-z_{\alpha}\right\}$ are pairwise disjoint and $\bigcup_{\alpha \in A}\left(G-z_{\alpha}\right)=$ $\boldsymbol{R}^{\infty}$. Hence there exists a countable set $\left\{\alpha_{i}\right\}_{i=1}^{\infty} \subseteq A$ such that $\alpha \in A \backslash\left\{\alpha_{i}\right\}_{i=1}^{\infty}$ implies $\mu\left(G-z_{\alpha}\right)=0$. If we let $\tilde{G}=G+\left\langle z_{\alpha_{i}}: 1 \leqq i<\infty\right\rangle$, it follows that $\mu(\widetilde{G}-z)=0$ for all $z \notin \widetilde{G}$.

Now, let $H_{n}=\tilde{G} \cap \boldsymbol{R}^{n}$. Since $G \in \mathscr{B}^{\infty}, H$ is a Borel set in $\boldsymbol{R}^{n}$, and hence $H_{n} \neq \boldsymbol{R}^{n}$ implies $m^{(n)}\left(H_{n}\right)=0$. Therefore there exists a sequence $\left\{y_{j}\right\}$ in $\boldsymbol{R}^{n} \backslash H_{n}$ such that $y_{j} \rightarrow 0$. Choose a compact set $K \subseteq \tilde{G}$ with $\mu(K)>0$. Then $\mu\left(K-y_{j}\right) \rightarrow \mu(K)>0$. Therefore there exists $r$ such that $0<\mu\left(K-y_{r}\right) \leqq$ $\mu\left(\widetilde{G}-y_{r}\right)$, a contradiction. We now have $\boldsymbol{R}^{n} \subseteq \widetilde{G}$. This implies that there exists $z \in\left\langle z_{\alpha_{i}}: 1 \leqq i<\infty\right\rangle$ such that $m^{(n)}\left((G-z) \cap \boldsymbol{R}^{n}\right)>0$. But then $m^{(n)}\left((G+z) \cap \boldsymbol{R}^{n}\right)>0$, since $(G-z) \cap \boldsymbol{R}^{n}=-\left[(G+z) \cap \boldsymbol{R}^{n}\right]$. Therefore $G \cap$ $\boldsymbol{R}^{n} \supseteq\left[(G+z) \cap \boldsymbol{R}^{n}\right]+\left[(G-z) \cap \boldsymbol{R}^{n}\right]$ contains an open set in $\boldsymbol{R}^{n}$. Hence 
$G \supseteq \boldsymbol{R}^{n}$. For $G \in \mathscr{B}_{\mu}^{\infty}$ with $\mu(G)>0$, choose a compact set $L \subseteq G$ with $\mu(L)>0$, and consider the $\mathscr{B}^{\infty}$-measurable subgroup $\langle L\rangle \leqq G$.

We record the last sentence of the proof as

COROLlary 1.1. Let $\mu$ be a regular, tight Borel measure on a Hausdorff topological group $X$. If $G$ is a completion measurable subgroup of $X$ with positive $\mu$-measure, there exists a $\sigma$-compact subgroup $H$ of $G$ with positive $\mu$-measure.

This fact will be used implicitly in the proofs of Theorem 2(b), (c), and (f).

CoRollary 1.2. $\mathscr{P} \subseteq \mathscr{E}_{1}$.

Proof. For $\mu \in \mathscr{P}$, let $\zeta_{n}=d \mu^{\pi} n / d m^{(n)}$. Then for $K$ compact

$$
\left|\mu^{\pi} n\left(\pi_{n} K\right)-\mu^{\pi}\left(\pi_{n} K-x\right)\right| \leqq \int\left|\zeta_{n}(y-x)-\zeta_{n}(y)\right| m^{(n)}(d y)
$$

for all $x \in \boldsymbol{R}^{n}$.

Therefore, since $K=\bigcap_{n=1}^{\infty} \pi_{n}^{-1}\left(\pi_{n}(K)\right)$ and $\mu \in \mathscr{P}$, we have, for $x \in \boldsymbol{R}^{N}$,

$$
|\mu(K-x)-\mu(K)| \leqq \int\left|\zeta_{.}(y-x)-\zeta_{. V}(y)\right| m^{\left(N^{\prime}\right)}(d y) .
$$

Hence $\mu(K-x) \rightarrow \mu(K)$ as $x \rightarrow 0$ in $\boldsymbol{R}^{x}$.

Corollary 1.3. If $\mu \in \mathscr{P}, G \leqq R^{\infty}$, and $G \in \mathscr{B}_{\mu}^{\infty}$, then $\mu(G)=0$ or 1 .

Proof. If $\mu(G)>0$, we have, $G \geqq l_{0}$, since $\mu \in \mathscr{E}_{1}$. But this implies that $G$ is a tail event. Hence $\mu(G)=1$.

Since in $\left(\boldsymbol{R}^{n}, \mathscr{B}^{n}, m^{(n)}\right)$ a measurable subgroup is either $\boldsymbol{R}^{n}$ or has Lebegue measure zero, the same is true for measures absolutely continuous with respect to $m^{(n)}$. In $\boldsymbol{R}^{\infty}$, if the tail $\sigma$-field of the coordinate mappings is a zero-one field for a measure $\mu$, and if a group contains $l_{0}$, then the group will have $\mu$-measure zero or one. In view of these facts a reasonable class of measures to consider is $\mathscr{E}_{2}$. In the theorem below we relate some of the properties of the $\mathscr{E}_{i}$.

THEOREM 2. (a) $\mathscr{P} \subseteq \mathscr{E}_{1} \cap \mathscr{E}_{2} \cap \mathscr{E}_{3}$. For $i=1,2$ :

(b) $\mu \in \mathscr{E}_{i}, \nu \ll \mu$ implies $v \in \mathscr{E}_{i}$.

(c) $\mu, v \in \mathscr{E}_{i}$, implies $\mu * v \in \mathscr{E}_{i}$.

(d) $\mathscr{E}_{i}$ is convex.

(e) $\mathscr{E}_{i}$ is closed in the total variation norm.

(f) $\mu_{a} \sim \mu$ for all $a \in l_{0}$ implies $\mu \in \mathscr{E}_{i}$.

(g) $\mathscr{E}_{3} \subseteq \mathscr{E}_{1} \cap \mathscr{E}_{2}$. 
(h) Suppose that $\varphi: \boldsymbol{R}^{\infty} \rightarrow \boldsymbol{R}^{\infty}$ is measurable, linear, and $\varphi\left(l_{0}\right)=l_{0}$. Then $\mu \in \mathscr{E}_{1}$ implies $\mu^{\varphi} \in \mathscr{E}_{1}$.

(i) $\mu * \mu \in \mathscr{E}_{1}$, implies $\mu \in \mathscr{E}_{1}$.

Proof. (a), (b), (d), (e), and (h) are clear.

(c) Let $\mu$ and $\nu \in \mathscr{E}_{1}$. Suppose that $\mu * \nu(G)>0$. Therefore there exists $x \in \boldsymbol{R}^{\infty}$ such that $\mu(G-x)>0$. Let $\tilde{G}=G+\langle x\rangle$. Then $\mu(\tilde{G})>0$ and hence $\tilde{G} \geqq l_{0}$. As in Theorem 1 this implies $G \geqq l_{0}$. The assertion for $\mathscr{E}_{2}$ is clear, since $(\mu * v)^{\pi_{n}}=\mu^{\pi_{n}} * v^{\pi_{n}}$.

(f) Suppose that $\mu_{a}$ and $\mu$ are mutually absolutely continuous for all $a \in l_{0}$, and $\mu(G)>0$. If $G \notin \boldsymbol{R}^{n}$, then $H_{n} \equiv G \cap \boldsymbol{R}^{n}$ has Lebesgue measure zero. Therefore there exists an uncountable number of distinct cosets $\left\{H_{n}-x_{\alpha}\right\}_{\alpha \in A}$ of $H_{n}$ in $\boldsymbol{R}^{n}$. But then $\left\{G-x_{\alpha}\right\}_{\alpha \in A}$ are distinct cosets of $G$ in $\boldsymbol{R}^{\infty}$. Since $\mu_{x_{\alpha}} \sim \mu$ for $\alpha \in A$, we have $\mu\left(G-x_{\alpha}\right)>0$ for all $\alpha \in A$, a contradiction. The assertion for $\mathscr{E}_{2}$ follows from the proof of Theorem 5 of [9].

(g) $\mathscr{E}_{3} \subseteq \mathscr{E}_{1}$ is the statement of Theorem 1. Let $\mu \in \mathscr{E}_{3}$, and let $C$ be a compact subset of $\boldsymbol{R}^{j}$. Given $\varepsilon>0$ there exists a compact set $K \subseteq \boldsymbol{R}^{\infty}$ such that $K \subseteq \pi_{j}^{-1}(C)$ and $\mu(K) \geqq \mu\left(\pi_{j}^{-1}(C)\right)-\varepsilon$. Let $\mu_{x}^{\pi_{j}}$ be defined on $\mathscr{B}^{j}$ by $\mu_{x}^{\pi_{j}}(A)=\mu^{\pi_{j}}(A-x)$. Since as $x \rightarrow 0$ in $\boldsymbol{R}^{j}, \mu_{x} \Rightarrow \mu$, we have

$$
\begin{aligned}
\mu\left(\pi_{j}^{-1}(C)\right) & \geqq \limsup _{x \rightarrow 0} \mu^{\pi_{j}}(C-x) \geqq \liminf _{x \rightarrow 0} \mu^{\pi_{j}}(C-x) \\
& \geqq \lim _{x \rightarrow 0} \mu(K-x)=\mu(K) \geqq \mu\left(\pi_{j}^{-1}(C)\right)-\varepsilon .
\end{aligned}
$$

Therefore

$$
\lim _{x \rightarrow 0} \mu^{\pi_{j}}(C-x)=\mu^{\pi_{j}}(C) .
$$

Now $\mu^{\pi_{j}}=v_{1}+v_{2}$ where $v_{1} \ll m^{(j)}, v_{2} \perp m^{(j)}$, and $v_{1} \perp v_{2}$. Hence $v_{2}(C-x) \rightarrow$ $v_{2}(C)$ as $x \rightarrow 0$ in $R^{j}$ for every compact set $C$. If $v_{2} \neq 0$, then there exists a compact set $L \subseteq \boldsymbol{R}^{j}$ such that $v_{2}(L)>0$ and $m^{(j)}(L)=0$; but then by Fubini's theorem we see that $v_{2}(L-x)=0$ for almost all $x\left[d m^{(j)}\right]$. Therefore $v_{2}(L)=0$, a contradiction. Therefore $\mu^{\pi_{j}} \ll m_{j}$.

(i) Suppose that $\mu * \mu \in \mathscr{E}_{1}$, and $\mu(G)>0$. Then

$$
\mu * \mu(G)=\int \mu(G-x) \mu(d x) \geqq \int_{G} \mu(G-x) \mu(d x)=\mu^{2}(G)>0 .
$$

Therefore $G \geqq l_{0}$. Hence $\mu \in \mathscr{E}_{1}$.

REMARKS. (1) In order to show $\mu * v \in \mathscr{E}_{1}$, we needed only that $\mu$ or $v \in \mathscr{E}_{1}$.

(2) Whenever one of the above properties holds for $\mathscr{E}_{2}$, it also holds for $\mathscr{E}_{1}$. It would be interesting, therefore, to prove or disprove " $\mathscr{E}_{2} \subseteq \mathscr{E}_{1}$ ".

(3) Most of the proofs in the above theorem hold if instead of $\boldsymbol{R}^{\infty}$, 
we consider the space $\prod_{i=1}^{\infty} G_{i}$, where $G_{i}$ is a connected, uncountable, abelian topological group with a regular Haar measure.

THEOREM 3. Let $\mu$ be a regular tight probability measure on a real Hausdorff topological vector space $X$, with nontrivial dual $X^{*}$. Suppose that there exists $\left\{\Lambda_{j}\right\} \subseteq X^{*}$ and $\left\{x_{j}\right\}_{j=1}^{\infty} \subseteq X$ such that:

(1) The tail $\sigma$-field of $\left\{\Lambda_{j}\right\}$ is a zero-one field for $\mu$.

(2) For $\mu$-almost all $x, x=\sum_{j=1}^{\infty} \Lambda_{j}(x) x_{j}$.

(3) If $\Gamma: X \rightarrow R^{\infty}$ is defined by $\Gamma(x)=\left(\Lambda_{j}(x)\right)_{j=1}^{\infty}$, then $\mu^{\Gamma} \in \mathscr{E}_{1}$.

Then if $G$ is a completion measurable subgroup of $X$, we have $\mu(G)=0$ or 1 .

PRoOF. Let $E=\left\{x \in X: x=\sum_{j=1}^{\infty} \Lambda_{j}(x) x_{j}\right\}$. Then $E$ is a subgroup of $X$ of $\mu$-measure 1. Now suppose that $G$ is a completion measurable subgroup of $X$ with positive $\mu$-measure. Since $\mu$ is regular and tight, we may choose a compact set $K$ contained in $E \cap G$ such that $\mu(K)>0$. Let $K_{0}=K \cup$ $(-K) \cup\{0\}$ and $K_{n+1}=K_{n}+K_{n}$. Then $H=\bigcup_{n=1}^{\infty} K_{n}$ is a subgroup of $G \cap E$ with positive $\mu$-measure. Since $\Gamma$ is continuous, $\Gamma(H)$ is $\sigma$-compact and hence measurable. But $\mu^{\Gamma}(\Gamma(H))>0$, and therefore $\Gamma(H) \geqq I_{0}$. Hence $\mu^{\mathrm{\Gamma}}(\Gamma(H))=1$ by (1). Since $H=E \cap \Gamma^{-1} \Gamma(H)$, we have that $\mu(G) \geqq \mu(H)=1$.

The next corollary follows from Theorem 3 and Theorem 2(f).

Corollary 3.1. Let $\mu \in \mathscr{M}$. Suppose that the following conditions hold:

(i) $\mu_{a} \sim \mu$ for all $a \in I_{0}$, and

(ii) the $\sigma$-field generated by the coordinate mappings is trivial with respect to $\mu$.

Then if $G \in \mathscr{B}_{\mu}^{\infty}$ and $G \leqq R^{\infty}$, we have $\mu(G)=0$ or 1 .

Proposition 3.2. Let $q: \boldsymbol{R}^{\infty} \rightarrow \boldsymbol{R}^{\infty}$ be linear and measurable, and let $\mu \in \mathscr{M}$. Suppose that for every $\mathscr{B}^{\infty}$-measurable subgroup $G$ of $\boldsymbol{R}^{\infty}$, we have $\mu(G)=0$ or 1 . Then for every $\mathscr{B}_{\mu^{q}}^{\infty}$-measurable subgroup $G$ of $\boldsymbol{R}^{\infty}$, we have $\mu^{q}(G)=0$ or 1 .

PRoof. By Corollary 1.1 we may consider $\mathscr{B}^{\infty}$-measurable subgroups. The rest of the proof is trivial.

In the next theorem we show that similar results hold for semigroups.

THEOREM 4. Let $\mu$ be a probability measure on a topological group $X$ such that:

(1) for every $A$ in the Borel $\sigma$-field, $\mu(A)=\mu\left(A^{-1}\right)$, where $A^{-1}=$ $\left\{a^{-1}: a \in A\right\}$, and

(2) every completion measurable subgroup of $X$ has $\mu$-measure 0 or 1 . Then if $X$ is a completion measurable subsemigroup of $X$ of $\mu$-measure greater than $\frac{1}{2}$, we have $\mu(S)=1$.

Proof. Let $S$ be as above. If $S^{-1}=\left\{x^{-1}: x \in S\right\}$, then $G=S \cap S^{-1}$ is a subgroup of $X$ of positive measure. Therefore $1=\mu(G) \leqq \mu(S)$. 
4. Examples. (a) Let $\mu$ be a mean-zero Gaussian measure on a real, separable Banach space $\left(B,\|\cdot\|_{B}\right)$. It is shown in [5] and [8] that there exists a Hilbert space, $H$, contained in $B$, such that $\mu$ is induced by the canonical normal distribution on $H$. If $\left\{\varphi_{j}\right\}_{j=1}^{\infty}$ is a complete orthonormal system for $H$ contained in $B^{*}$, then for $\mu$-almost all $x$ in $B$,

$$
\lim _{N}\left\|x-\sum_{j=1}^{N}\left(x, \varphi_{j}\right) \varphi_{j}\right\|_{B}=0
$$

(see [3] and [7]). Since $\left\{\left(x, \varphi_{j}\right)\right\}_{j=1}^{\infty}$ is a sequence of independent Gaussian random variables with mean zero and variance $\left\|\varphi_{j}\right\|_{H}^{2}=1$, the hypotheses of Theorem 3 are satisfied.

In this case we can even say more.

THEOREM 5. In the above setting $H=\bigcap G$, where the intersection is taken over all completion measurable subgroups, $G$, of positive $\mu$-measure.

Proof. As before let $\Gamma: B \rightarrow \boldsymbol{R}^{\infty}$ be defined by $\Gamma(x)=\left(\left(x, \varphi_{j}\right)\right)_{j=1}^{\infty}$, and $E=\left\{x \in B: \lim _{N}\left\|x-\sum_{j=1}^{N}\left(x, \varphi_{j}\right) \varphi_{j}\right\|_{B}=0\right\}$. Since $\left(\mu^{\Gamma}\right)_{\alpha} \sim \mu^{\Gamma}$ if and only if $\alpha \in l_{2}$, we see by the proof of Theorem 2(f), that any $\mathscr{B}_{\mu}^{\infty}$-measurable subgroup, $G$, of $\boldsymbol{R}^{\infty}$ with $\mu^{\Gamma}(G)>0$, contains $l_{2}$.

Now, for any $\gamma \in \boldsymbol{R}^{\infty}$, let $M_{\gamma}=\left\{x \in \boldsymbol{R}^{\infty}:\left|\sum_{i=1}^{\infty} \gamma_{i} x_{i}\right|<\infty\right\}$. It is easy to see that $\mu^{\Gamma}\left(M_{\gamma}\right)>0$ if and only if $\gamma \in l_{2}$. Let $M=\bigcap_{\gamma \in l_{2}} M_{\gamma}$. By the BanachSteinhaus theorem $M=l_{2}$. However,

$$
\bigcap_{\gamma \in l_{2}} \Gamma^{-1}\left(M_{\gamma}\right) \cap E=\Gamma^{-1}\left(l_{2}\right) \cap E=H .
$$

Hence we are done.

(b) Let $\left\{\xi_{j}\right\}_{j=1}^{\infty}$ be a sequence of independent random variables with densities, and let $\mu$ be the measure on $\boldsymbol{R}^{\infty}$ induced by $\left\{\xi_{j}\right\}_{j=1}^{\infty}$. Now fix a nonnegative integer $m$ and real numbers $a_{0}, \cdots, a_{m}$ (not all zero). Let

$$
\zeta_{j}=\sum_{i=1}^{m+1} a_{i-1} \xi_{j+m+1-i}
$$

for $j \geqq 1$, and $\nu$ the measure on $\boldsymbol{R}^{\infty}$ induced by $\left\{\zeta_{j}\right\}_{j=1}^{\infty}$. Define $\varphi: \boldsymbol{R}^{\infty} \rightarrow \boldsymbol{R}^{\infty}$ by

$$
[\varphi(x)]_{j}=\sum_{i=1}^{m+1} a_{i-1} x_{j+m+1-i} .
$$

It is easy to see that $\varphi\left(l_{0}\right)=l_{0}$ and $\nu=\mu^{\varphi}$. Hence any $\mathscr{B}_{v}^{\infty}$-measurable subgroup of $\boldsymbol{R}^{\infty}$ with positive $\boldsymbol{v}$-measure contains $l_{\mathbf{0}}$. Hence by Proposition 3.2 , any $\mathscr{B}_{v}^{\infty}$-measurable subgroup has $v$-measure zero or one.

(c) Let $\left\{\xi_{j}\right\}_{j=1}^{\infty}$ be a Markov chain with state space the real line, and let $\mu$ be the measure on $\boldsymbol{R}^{\infty}$ induced by $\left\{\xi_{j}\right\}_{j=1}^{\infty}$. Assume that $\mu \in \mathscr{E}_{2}$ and 
the tail $\sigma$-field is trivial. Then using an argument similar to that of Corollary 1.2 , we can show that $\mu \in \mathscr{E}_{3}$, and hence $\mu \in \mathscr{E}_{1}$. Therefore, since the tail $\sigma$-field is trivial, measurable subgroups satisfy the zero-one law with respect to $\mu$.

ACKNOWLedgment. The author wishes to thank Professor J. D. Kuelbs for suggesting this problem, and for his helpful comments.

\section{REFERENCES}

1. R. H. Cameron and R. E. Graves, Additive functionals on a space of continuous functions. I, Trans. Amer. Math. Soc. 70 (1951), 160-176. MR 12, 718.

2. N. C. Jain, A zero-one law for Gaussian processes, Proc. Amer. Math. Soc. 29 (1971), 585-587. MR 43 \#4099.

3. N. C. Jain and G. Kallianpur, Norm convergent expansions for Gaussian processes in Banach spaces, Proc. Amer. Math. Soc. 25 (1970), 890-895. MR 42 \#1211.

4. B. Jamison and S. Orey, Subgroups of sequences and paths, Proc. Amer. Math. Soc. 24 (1970), 739-744. MR 40 \#121.

5. G. Kallianpur, Abstract Wiener processes and their reproducing kernel Hilbert spaces, Z. Wahrscheinlichkeitstheorie und Verw. Gebiete 17 (1971), 113-123. MR 43 \#6961.

6. _- Zero-one laws for Gaussian processes, Trans. Amer. Math. Soc. 149 (1970), 199-211. MR 42 \#1200.

7. J. Kuelbs, Expansions of vectors in a Banach space related to Gaussian measures, Proc. Amer. Math. Soc. 27 (1971), 364-370. MR 42 \#2517.

8. _ Gaussian measures on a Banach space, J. Functional Analysis 5 (1970), 354-367. MR 41 \#4639.

9. A. V. Skorohod, Admissible shifts of measures in Hilbert space, Teor. Verojatnost. i Primenen. 15 (1970), 577-598=Theor. Probability Appl. 15 (1970), 557-580. MR 45 \#499.

School of Mathematics, University of Minnesota, Minneapolis, Minnesota 55455

Current address: Department of Mathematics and Statistics, University of Massachusetts, Amherst, Massachusetts 01002 